\title{
Taste classification of foods consumed in the National Diet and Nutrition Survey
}

\author{
A. O. Bawajeeh ${ }^{1,2}$, M. A. Zulyniak ${ }^{1}$, C. E. Evans ${ }^{1}$ and J. E. Cade ${ }^{1}$ \\ ${ }^{1}$ Nutritional Epidemiology Group, School of Food Science and Nutrition, University of Leeds, Leeds LS2 9JT, UK and \\ ${ }^{2}$ Department of Food and Nutrition, Faculty of Human Sciences and Design, King Abdulaziz University, \\ Jeddah 3270, Saudi Arabia
}

Taste of food may be important in food choice and dietary intakes ${ }^{(1,2)}$. While taste perception in the laboratory has been widely studied, it has rarely been taken into account when exploring dietary patterns in a population ${ }^{(3)}$. The aim of this work is to identify consumers' taste perceptions for a list of foods.

The National Diet and Nutrition Survey (NDNS) rolling programme year 9 was used to generate a list of foods consumed by adolescents (10-19 years old). 1748 different foods were grouped by type/food group, and/or sugar/salt contents into 7 main and 23 subgroups. A pilot test identified foods with high agreement to further limit the list. The shortened food list was included in an online survey which was distributed to Facebook groups and Twitter. Food-taste classification questions asked participants to allocate one main taste to each food: sweet, salty, sour, bitter and savoury/umami; neutral and never tried. To minimize participant burden, the food list was divided into 3 and participants were asked to complete one part with an option to complete the rest. Foods were allocated to taste categories based on agreement by $\geq 50 \%$ of respondents. If there was a $<50 \%$ agreement, foods were classified by allocation to two+ taste categories based on the highest scoring taste combinations

The pilot questionnaire was completed by 19 participants. $15 / 19$ rated 55 foods as the same taste and these were removed from the final questionnaire. The final food list contained 184 items.

The main online questionnaire obtained 209 responses from 162 females, 44 males, ( 3 not known). The age range was from 18-70+ years with the majority between 40-59 years old. Seven reported having COVID-19, but only 3 were tested positive (none experienced a taste loss). Food items were classified as 29\% neutral, $22 \%$ sweet, $11 \%$ savoury/umami, $9 \%$ salty, $7 \%$ bitter, and $5 \%$ sour. Six mixed tastes categories were identified: savoury/umami-salty (11\%), sweet-sour (3\%), sweet-bitter (2\%), sour-bitter (1\%), salty-bitter ( $0.5 \%)$ and savoury/umami-bitter (1\%). In the next step of the study, taste categories will be applied to the remaining foods consumed by adolescents reported in the NDNS to generate taste patterns allowing us to explore links with nutrition and anthropometry.

We have characterised major tastes for all foods consumed in the most recent NDNS. This will allow us to create a UK food-taste database and explore dietary-taste patterns in the NDNS.

\section{References}

1. Ensaff H, Coan S, Sahota P et al. (2015) Nutrients 7, 4619-37.

2. Hallstrom L, Vereecken C, Ruiz J et al. (2011) Appetite 56, 649-57.

3. van Langeveld A, Teo P, de Vries et al. (2018) Br. J. Nutr 1195-206. 